VOL. 60 (1999) [81-94]

\title{
QUASI-CONCAVE FUNCTIONS AND CONVEX CONVERGENCE TO INFINITY
}

\author{
Gerald BEer
}

\begin{abstract}
By a convex mode of convergence to infinity $\left\langle C_{k}\right\rangle$, we mean a sequence of nonempty closed convex subsets of a normed linear space $X$ such that for each $k$, $C_{k+1} \subset \operatorname{int} C_{k}$ and $\bigcap_{k=1}^{\infty} C_{k}=\emptyset$, and a sequence $\left\langle x_{n}\right\rangle$ is $X$ is declared convergent to infinity with respect to $\left\langle C_{k}\right\rangle$ provided each $C_{k}$ contains $x_{n}$ eventually. Positive convergence to infinity with respect to a pointed cone with nonempty interior as well as convergence to infinity in a fixed direction fit within this framework. In this paper we study the representation of convex modes of convergence to infinity by quasi-concave functions and associated remetrizations of the space.
\end{abstract}

\section{INTRODUCTION}

Everyone agrees as to what positive convergence to infinity for a sequence $\left\langle x_{n}\right\rangle$ in Euclidean space means: for each fixed $x$, eventually $x \leqslant x_{n}$ with respect to the usual componentwise partial order. Put differently, $\left\langle x_{n}\right\rangle$ converges positively to infinity if eventually $x_{n} \in x+P$ where $P$ is the cone of nonnegative vectors. Given a closed pointed cone $C$ for a normed linear space $X$ with nonempty interior, it is thus natural to declare a sequence $\left\langle x_{n}\right\rangle$ in $X$ positively convergent to infinity if given any $x \in X$ we have $x_{n} \in x+C$ eventually. If we fix $x_{0} \in \operatorname{int} C$, then $\left\langle x_{n}\right\rangle$ is positively convergent to infinity if and only if for each $k \in Z^{+}$, we have $x_{n} \in k x_{0}+C$ eventually. Clearly, this condition is independent of the choice of the point $x_{0}$.

Abstracting from this situation, suppose $\left\langle A_{k}\right\rangle$ is a sequence of nonempty closed subsets of a (noncompact) metrisable space $X$ such that for each $k, A_{k+1} \subset \operatorname{int} A_{k}$ and $\bigcap_{k=1}^{\infty} A_{k}=\emptyset$ (note that these sets could all be bounded). We declare a sequence $\left\langle x_{n}\right\rangle$ convergent to infinity relative to $\left\langle A_{k}\right\rangle$ if each $A_{k}$ contains $x_{n}$ eventually. Two such sequences $\left\langle A_{k}\right\rangle$ and $\left\langle B_{k}\right\rangle$ will be declared equivalent - and we write $\left\langle A_{k}\right\rangle \equiv\left\langle B_{k}\right\rangle$ - provided for each $k \in Z^{+}$there exists $n \in Z^{+}$and $j \in Z^{+}$such that $A_{k} \supset B_{n}$ and $B_{k} \supset A_{j}$, and the induced equivalence classes will be called modes of convergence to infinity [2]. Convergence of a sequence $\left\langle x_{n}\right\rangle$ to infinity for such an equivalence class is

Received 24th December, 1998

Copyright Clearance Centre, Inc. Serial-fee code: 0004-9727/99 \$A2.00+0.00. 
independent of representative, and in the sequel we shall freely engage in the abuse of identifying equivalence classes with their representatives.

One of the first modes of convergence to infinity that comes to mind is convergence to infinity in distance with respect to an admissible unbounded metric $\rho$, where fixing a point $x_{0} \in X$, we take $A_{k}=\left\{x \in X: \rho\left(x, x_{0}\right) \geqslant k\right\}, k=1,2,3, \ldots$ It is a remarkable yet simple fact that all modes of convergence to infinity arise in this way. Given a mode of convergence to infinity $\left\langle A_{k}\right\rangle$, declare a subset $B$ of $X$ bounded with respect to $\left\langle A_{k}\right\rangle$ provided for some $k \in Z^{+}$, we have $B \cap A_{k}=\emptyset$. It is easy to check that the bounded sets so defined satisfy all the properties that one expects that the bounded subsets of a metric space satisfy [2, Theorem 3.2]. Using Urysohn functions [12, p.189], one can construct a continuous nonnegative function $\varphi: X \rightarrow \mathbf{R}$ such that $\sup _{x \in B} \varphi(x)<\infty$ if and only if $B$ is bounded with respect to $\left\langle A_{k}\right\rangle$. Then starting with an arbitrary metric $d$ for $X$, it is easily seen that $\rho(x, y)=\min \{d(x, y), 1\}+|\varphi(x)-\varphi(y)|$ is an admissible metric for $X$ and that the $\rho$-bounded sets coincide with the sets that are bounded with respect to $\left\langle A_{k}\right\rangle[12, \mathrm{p} .191]$, which is to say that $\left\langle A_{k}\right\rangle \equiv\left\{x \in X: \rho\left(x, x_{0}\right) \geqslant k\right\}[2$, Corollary 3.3].

We call a function $\varphi$ as described above a forcing function for $\left\langle A_{k}\right\rangle$. Evidently. $\varphi$ is a forcing function for $\left\langle A_{k}\right\rangle$ if and only if $\langle\{x: \varphi(x) \geqslant k\}\rangle \equiv\left\langle A_{k}\right\rangle$, and a sequence $\left\langle x_{n}\right\rangle$ in $X$ is convergent to infinity with respect to $\left\langle A_{k}\right\rangle$ if and only if $\lim _{n \rightarrow \infty} \varphi\left(x_{n}\right)=\infty$ [2]. In the case of positive convergence to infinity in $\mathbf{R}^{n}$ a natural forcing function exists: $\varphi\left(\alpha_{1}, \alpha_{2}, \ldots, \alpha_{n}\right)=\max \left\{0, \min \left\{\alpha_{1}, \alpha_{2}, \ldots, \alpha_{n}\right\}\right\}$. In the case of convergence in distance with respect to an unbounded metric $\rho$ for a metrisable space $X$, a forcing function is $\varphi(x)=\rho\left(x, x_{0}\right)$ where $x_{0}$ is a fixed point of $X$. Often, a mode of convergence to infinity is most easily described in forcing function form. For example, in the Euclidean plane, the following forcing function represents positive convergence to infinity, along with the condition that $\alpha_{1}=o\left(\alpha_{2}^{2}\right): \varphi\left(\alpha_{1}, \alpha_{2}\right)=\min \left\{\alpha_{1}, \alpha_{2}, \alpha_{2}^{2} / \alpha_{1}\right\}$ when $\alpha_{1}>0$ and $\alpha_{2}>0$ and $\varphi\left(\alpha_{1}, \alpha_{2}\right)=0$ otherwise.

Returning to the context of a normed linear space $X$, we call a mode of convergence to infinity convex if it has a representative $\left\langle C_{k}\right\rangle$ consisting of convex sets. The purpose of this article is to show that such modes of convergence to infinity are those that admit quasi-concave forcing functions, and we show that a uniformly continuous quasi-concave forcing function exists if and only if a Lipschitz quasi-concave forcing function exists. Special attention is given to the case of positive convergence to infinity as determined by a cone with nonempty interior.

\section{TERMINOLOGY AND NOTATION}

Throughout, $X$ will be a normed linear space with origin $\theta$ and closed unit ball $U$. Its continuous dual $X^{*}$ will have origin $\theta^{*}$, and closed unit ball $U^{*}$. We denote 
the set of norm-one elements of $X^{*}$ by $S^{*}$.

A function $f: X \rightarrow(-\infty, \infty]$ is called convex if $\{(x, \alpha): x \in X, \alpha \in \mathbf{R}$ and $\alpha \geqslant$ $f(x)\}$ is a convex subset of $X \times \mathbf{R}$, and quasi-convex if for each $\alpha \in \mathbf{R}$, the sublevel set $\{x \in X: f(x) \leqslant \alpha\}$ is a convex subset of $X$. We call a function $f$ concave (respectively, quasi-concave) provided $-f$ is convex (respectively, quasi convex). If $A$ is a convex subset of $X$, there are three basic convex functions associated with it. The distance functional $d(\cdot, A)$ for $A$ is defined by $d(x, A)=\inf _{a \in A}\|x-a\|$. The indicator function $I(\cdot, A)$ for $A$ is defined by

$$
I(x, A)= \begin{cases}0 & \text { if } x \in A \\ \infty & \text { if } x \notin A .\end{cases}
$$

The support functional $s(\cdot, A): X^{*} \rightarrow(-\infty, \infty]$ for $A$ is defined by $s(y, A)=\sup _{a \in A} y(a)$. The polar $A^{\circ}$ of $A$ is defined by $A^{\circ}=\left\{y \in X^{*}: s(y, A) \leqslant 1\right\}$.

By a cone $C$ in $X$ we mean a convex subset of $X$ closed under multiplication by nonnegative scalars. For example, if $A$ is a closed convex set, then $\{c \in X: a+\lambda c \in$ $A$ for some $a \in A$ and all $\lambda>0\}$ is a closed cone called the recession cone of $A$. A cone $C$ is called pointed if $C \cap-C=\theta$. Given pointed cone $C$, the relation $x \leqslant y$ provided $y-x \in C$ defines a partial order on $X$, and $C$ is called the positive cone with respect to this order. We denote the set of positive linear functionals on $X$ by $C^{*}$ :

$$
C^{*}=\left\{y \in X^{*}: \forall x \in C, y(x) \geqslant 0\right\}
$$

This set is a weak ${ }^{*}$-closed cone in $X^{*}$. Clearly, $C^{*}=-C^{\circ}=(-C)^{\circ}$, and by the Bipolar Theorem

$$
C=\left\{x \in X: \forall y \in C^{*}, y(x) \geqslant 0\right\}
$$

provided $C$ is closed (see, for example, $[4$, p.222]).

\section{Distance Functionals For CONVEX SETS AND THEIR COMPLEMENTS}

A well-known formula in convex analysis gives a dual description of the distance $d(x, A)$ from a point $x$ in a normed linear space $X$ to a closed convex set $A$ in terms of the values of the support functional $s(\cdot, A)$ for the set $A$ :

$$
d(x, A)=\sup \left\{y(x)-s(y, A): y \in U^{*}\right\} .
$$

Since the dual unit ball $U^{*}$ is weak ${ }^{*}$ compact and $y \in y(x)-s(y, A)$ is weak ${ }^{*}$ upper semicontinuous, the "sup" can be replaced by "max". Although this result follows directly from the separation theorem (see, for example $[1, p .30]$ ), there are other ways to prove this using more elaborate machinery. One proof is based on the representation 
of the distance function $d(\cdot, A)$ as the epi-sum (infimal convolution) of the norm of $X$ and indicator function $I(\cdot, A)$ of the set $A$, and since the conjugate of an epi-sum is the sum of the conjugates, it is easily seen that the above formula is nothing but the second conjugate of $d(\cdot, A)$ evaluated at $x[11, \mathrm{p} .62]$. A second proof can be given using the Fenchel-Moreau-Rockafellar Duality Theorem [16, pp.106-107]. Yet a third proof based on Sion's minimax theorem [15] has been communicated to me by S. Simons: since $U^{*}$ is weak ${ }^{*}$ compact and $(a, y) \rightarrow y(x-a)$ on $A \times U^{*}$ is continuous and convex in $a$ and weak ${ }^{*}$-continuous and concave in $y$, we have

$$
\begin{aligned}
\max _{y \in U^{*}} y(x)-s(y, A) & =\max _{y \in U^{*}} \inf _{a \in A} y(x)-y(a) \\
& =\inf _{a \in A} \max _{y \in U^{*}} y(x-a) \\
& =\inf _{a \in A}\|x-a\| .
\end{aligned}
$$

Still, this dual formula - in essence - is misleading. There are really just two possibilities for a point $x \in X$ relative to $A$ : either $x \in A$, where the max is attained using the zero element $\theta^{*} \in U^{*}$, or $x \notin A$, where the $\max$ is attained using a norm one element $y$ of $X^{*}$. Thus, for $x \in A^{c}$, we have

$$
d(x, A)=\sup _{y \in S^{*}} y(x)-s(y, A) .
$$

Now suppose $A$ is a closed convex body, that is, int $A \neq \emptyset$, and also that $A \neq X$. If $x_{1}+r_{1} U$ and $x_{2}+r_{2} U$ are contained in $A$ and $\lambda \in(0,1)$, then

$$
\lambda x_{1}+(1-\lambda) x_{2}+\left[\lambda r_{1}+(1-\lambda) r_{2}\right] U \subset A
$$

which shows that $d\left(\cdot, A^{c}\right)$ is a concave function on $A$. But this, too, can be represented dually. It is a straight-forward exercise to show that for $x \in A$,

$$
d\left(x, A^{c}\right)=\inf _{y \in S^{*}} s(y, A)-y(x)
$$

$[6,13]$, and as a result, the distance to the boundary of a closed convex body is the absolute value of the convex function $\Delta(\cdot, A): X \rightarrow \mathbf{R}$

$$
\Delta(x, A)=\sup _{y \in S^{*}} y(x)-s(y, A)=d(x, A)-d\left(x, A^{c}\right) .
$$

As a supremum of 1-Lipschitz functions, $\Delta$ is 1-Lipschitz, too. The function $\Delta(\cdot, A)$ was introduced by Hirart-Urruty in $[8,9]$, where he showed that $\Delta$ can be written as the infimal convolution of the norm with $-d\left(\cdot, A^{c}\right)+I(\cdot, A)$.

There is one aspect in which the formulas that represent $d(\cdot, A)$ and $d\left(\cdot, A^{c}\right)$ for a convex body $A$ are not mirror images of one another: whereas in the former case, the "sup" is a "max", in the latter, the "inf" need not be a "min", even in Hilbert space. 
Example. Let $A=\left\{x \in \ell_{2}: \forall n \in Z^{+}|x(n)| \leqslant 1+1 / n\right\}$. Notice that $A$ is an unbounded closed convex set containing the closed unit ball. Let $\left\{e_{n}: n \in Z^{+}\right\}$be the usual orthonormal set of vectors in $\ell_{2}$. Since $b d A=\{x \in A$ : for some $n,|x(n)|=$ $1+1 / n\}$. it is clear that $d\left(\theta, A^{c}\right)=d(\theta, b d A)=1$. We claim that for each norm one element $y$ of $\ell_{2}^{*}=\ell_{2}$, we have $s(y, A)-y(\theta)=s(y, A)>1$. If $y=e_{n}$ for some $n$, then

$$
s(y, A) \geqslant y\left(\frac{n+1}{n} e_{n}\right)=\frac{n+1}{n}>1
$$

and similarly if $y=-e_{n}$ for some $n$, then $s(y, A)>1$. Otherwise, there exists $\delta>0$ such that for each $n,|y(n)|<1-\delta$. Define $w \in \ell_{2}$ by

$$
w(n)= \begin{cases}\delta / n & \text { if } y(n) \geqslant 0 \\ -\delta / n & \text { if } y(n)<0\end{cases}
$$

Then $y+w \in A$ and $y(w)>0$ so that $s(y, A) \geqslant y(y+w)=\|y\|^{2}+y(w)>1$.

Sublevel sets of the distance functional $d(\cdot, A)$ for a (convex) set $A$ are called outer parallel bodies of $A$. Formally, if $\lambda \geqslant 0$, we shall write $B_{\lambda}(A)$ for $\{x \in X$ : $d(x, A) \leqslant \lambda\}$. Of course, in a general Banach space the inclusion $A+\lambda U \subset B_{\lambda}(A)$ may be proper; the sets $A+\lambda U$ are often called enlargements of $A$. Following standard practice in finite dimensions $[\mathbf{7}, \mathbf{1 4}]$, if $A$ is a closed convex body in a normed linear space $X$ with $A \neq X$, we call the superlevel sets of the concave functional $d\left(\cdot, A^{c}\right)$ restricted to $A$ inner parallel bodies of the set $A$. For notation, if $\lambda \geqslant 0$, we write

$$
B_{-\lambda}(A)=\left\{x \in A: d\left(x, A^{c}\right) \geqslant \lambda\right\} .
$$

In the case of inner parallel bodies, the result will be empty if $\lambda$ exceeds the inradius of the set if that happens to be finite. With respect to Hiriart-Urruty's functional we have the following simple, attractive formula that we shall use in Section 5:

$$
B_{\lambda}(A)=\{x \in X: \Delta(x, A) \leqslant \lambda\} \quad(\lambda \in \mathbf{R}) .
$$

Although we shall not use them, we note representation formulas for outer and inner parallel bodies which say that an outer (respectively inner) parallel body of radius $\lambda$ can be obtained by moving each of the bounding hyperplanes that define the convex set $A$ out (respectively, in) by a distance $\lambda \geqslant 0$ :

$$
\begin{aligned}
B_{\lambda}(A) & =\left\{x \in X: \forall y \in S^{*}, y(x) \leqslant s(y, A)+\lambda\right\}, \\
B_{-\lambda}(A) & =\left\{x \in X: \forall y \in S^{*}, y(x) \leqslant s(y, A)-\lambda\right\} .
\end{aligned}
$$

These follow easily from the duality formulas given above. 


\section{FORCING FUNCTIONS FOR CONVEX MODES OF CONVERGENCE TO INFINITY}

Let $\left\langle C_{k}\right\rangle$ be a sequence of closed convex ses in a normed linear space $X$ such that for each $k, C_{k+1} \subset \operatorname{int} C_{k}$ and $\bigcap_{k=1}^{\infty} C_{k}=\emptyset$. It is not in general possible to find a forcing $\varphi$ for $\left\langle C_{k}\right\rangle$ whose restriction to any $C_{k}$ is concave. To see this, recall that $\varphi$ is a forcing function for $\left\langle C_{k}\right\rangle$ if and only if for each $k \in Z^{+}$, there exist integers $j$ and $n$ such that $C_{k} \supset\{x: \varphi(x) \geqslant n\}$ and $\{x: \varphi(x) \geqslant k\} \supset C_{j}$. This in turn forces the sets $C_{k}$ to have the common recession cone shared by the superlevel sets of $\varphi[10$, p.180], which may not a priori be the case. Here is a practical example.

EXAMPLE. Let $x_{0}$ be a norm one vector in $X$, and choose $y_{0} \in S^{*}$ with $y_{0}\left(x_{0}\right)=1$. Then convergence to infinity in the direction $x_{0}$ is determined by the sequence of closed convex sets $\left\langle K_{n} \cap\left\{x \in X: y_{0}(x) \geqslant n\right\}\right\rangle$ where $K_{n}$ is the closure of the set of all positive multiples of elements $x_{0}+U / 2 n$. There is no compatible concave forcing function, as the recession cones shrink to a single ray.

Evidently, each mode of convergence to infinity that admits a quasi-concave forcing function $\varphi$ is convex, for the sequence of convex sets $\langle\{x: \varphi(x) \geqslant k\}\rangle$ then represents the mode of convergence. Although the search for a concave forcing function is in general futile, we aim to show that each convex mode of convergence to infinity $\left\langle C_{k}\right\rangle$ always admits a quasi-concave forcing function.

Lemma 4.1. Let $X$ be a normed linear space, and let $A \neq X$ be a closed convex body in $X$. Suppose $C$ is a closed convex set with $C \subset \operatorname{int} A$. Then there exists a closed convex body $B$ with $C \subset$ int $B \subset B \subset$ int $A$.

Proof: The function $f: A \rightarrow \mathbf{R}$ defined by $f(x)=d(x, C)-d\left(x, A^{c}\right)$ is continuous and convex. If $x \in C$, then $x \in \operatorname{int} A$ and so $f(x)<0$. If $x \in b d A$, then $x \notin C$ and so $f(x)>0$. As a result, $B=\{x \in A: f(x) \leqslant 0\}$ does the job.

Proposition 4.2. Let $X$ be a normed linear space, and let $A \neq X$ be a closed convex body in $X$. Suppose $C$ is a closed convex set with $C \subset \operatorname{int} A$. Then there exists a quasi-concave continuous function $f: X \rightarrow[0,1]$ such that $f\left((\operatorname{int} A)^{c}\right)=0$ and $f(C)=1$.

PROOF: We repeat the essential construction of Uryshohn's Lemma, using Lemma 4.1 above. with a special twist for the enjoyment of unilateral analysis enthusiasts. We first take a closed convex body $B_{1 / 2}$ such that

$$
C \subset \operatorname{int} B_{1 / 2} \subset B_{1 / 2} \subset \operatorname{int} A \text {. }
$$

Then we choose closed bodies $B_{1 / 4}$ and $B_{3 / 4}$ such that

$$
C \subset \operatorname{int} B_{3 / 4} \subset B_{3 / 4} \subset \operatorname{int} B_{1 / 2}
$$


and

$$
B_{1 / 2} \subset \operatorname{int} B_{1 / 4} \subset B_{1 / 4} \subset \operatorname{int} A \text {. }
$$

Continuing our insertions, for each dyadic rational $r \in(0,1)$ we produce a closed convex body $B_{r}$ such that whenever $r<p$, then

$$
C \subset \operatorname{int} B_{p} \subset B_{p} \subset \operatorname{int} B_{r} \subset B_{r} \subset \operatorname{int} A .
$$

Define $f: X \rightarrow[0,1]$ by $f(x)=\sup \left\{r: x \in \operatorname{int} B_{r}\right\}$ if $x$ belongs to some $B_{r}$ and $f(x)=0$ otherwise. Since $C$ lies inside each $B_{r}$ we have $f(C)=1$, and since (int $\left.A\right)^{c}$ meets no set $B_{r}$ we have $f\left((\operatorname{int} A)^{c}\right)=0$. Also, define $g: X \rightarrow[0,1]$ by $g(x)=\inf \{s$ : $\left.x \notin \operatorname{int} B_{s}\right\}$ if $x$ fails to belong to some $B_{s}$ and $g(x)=1$ otherwise. We leave it to the reader to show that $f=g$ at each point of $X$. Continuity of $f$ follows from the lower semicontinuity of $f$ plus the upper semicontinuity of $g$, properties that should be obvious from the formula

$$
f(x)=\sup _{0<r<1} f_{r}(x)
$$

where for each dyadic rational $r \in(0,1)$, the function $f_{r}$ is $r$ times the characteristic function of int $B_{r}$, and

$$
g(x)= \begin{cases}1 & \text { if } x \in \bigcap B_{s} \\ \inf _{0<s<1} g_{s}(x) & \text { otherwise }\end{cases}
$$

where for each dyadic rational $s \in(0,1)$, the function $g_{s}$ is $s$ times the characteristic function of (int $\left.B_{s}\right)^{c}$. Finally, for each $\alpha \in(0,1],\{x: f(x) \geqslant \alpha\}=\bigcap_{r<\alpha} \operatorname{int} B_{r}$ which is a convex set, and so $f$ is quasi-concave.

THEOREM 4.3. Suppose $\left\langle C_{k}\right\rangle$ is a sequence of nonempty closed convex subsets of a normed linear $X$ such that for each $k, C_{k+1} \subset \operatorname{int} C_{k}$ and $\bigcap_{k=1}^{\infty} C_{k}=\emptyset$. Then there exists a quasi-concave forcing function $\varphi$ for $\left\langle C_{k}\right\rangle$.

Proof: Without loss of generality, we may assume that $C_{1}$ is a proper subset of $X$. By Proposition 4.2, for each $k \in Z^{+}$there is a continuous quasi-concave function $f_{k}: X \rightarrow[k-1, k]$ such that

$$
f_{k}(x)= \begin{cases}k-1 & \text { if } x \in\left(\operatorname{int} C_{k}\right)^{c} \\ k & \text { if } x \in C_{k+1}\end{cases}
$$

Now define a nonnegative function $\varphi$ on $X$ by

$$
\varphi(x)= \begin{cases}f_{1}(x) & \text { if } x \in\left(\operatorname{int} C_{2}\right)^{c} \\ f_{k}(x) & \text { if } x \in C_{k} \cap\left(\operatorname{int} C_{k+1}\right)^{c} \text { for } k \geqslant 2 .\end{cases}
$$


Since $f_{k}(x)=f_{k+1}(x)=k$ whenever $x \in b d C_{k+1}, \varphi$ is well-defined. Now the family

$$
\left\{\left(\text { int } C_{2}\right)^{c}\right\} \cup\left\{C_{k} \cap\left(\operatorname{int} C_{k+1}\right)^{c}: k=2,3,4, \ldots\right\}
$$

forms a locally finite closed cover of $X$, and as the restriction of $\varphi$ to each closed set in the family is continuous, $\varphi$ is globally continuous $[5$, p.83]. Furthermore, $\varphi$ is quasi-concave, for if $\alpha \leqslant 0$, then $\{x: \varphi(x) \geqslant \alpha\}=X$, and if $\alpha>0$, then $\{x: \varphi(x) \geqslant \alpha\}=\left\{x: f_{k}(x) \geqslant \alpha\right\}$ where $k$ is the smallest integer such that $k \geqslant \alpha$. Finally, if $k$ is a positive integer, then $C_{k} \supset\{x: \varphi(x) \geqslant k\} \supset C_{k+1}$, from which it follows that $\varphi$ is a forcing function for $\left\langle C_{k}\right\rangle$.

We now turn to necessary and sufficient conditions for the existence of a Lipschitz continuous quasi-concave forcing function for a convex mode of convergence to infinity. The result we give is anticipated by [2, Theorem 4.2] that is valid in general metric space: However, the construction of the forcing function therein involves a sum of functions. and quasi-concavity is not preserved by sums.

THEOREM 4.4. Suppose $\left\langle C_{k}\right\rangle$ is a sequence of nonempty closed convex subsets of a normed linear $X$ such that for each $k, C_{k+1} \subset \operatorname{int} C_{k}$ and $\bigcap_{k=1}^{\infty} C_{k}=\emptyset$. The following conditions are equivalent:

(1) $\left\langle C_{k}\right\rangle$ admits a uniformly continuous quasi-concave forcing function $\varphi$;

(2) there exists $\delta>0$ such that for all $k \in Z^{+}$there exists $n>k$ with $C_{k} \supset C_{n}+\delta U$

(3) for all $k \in Z^{+}$and $r>0, B_{-r}\left(C_{k}\right)$ contains $C_{n}$ for all sufficiently large $n$;

(4) $\left\langle C_{k}\right\rangle$ admits a Lipschitz continuous quasi-concave forcing function $\varphi$.

ProOF: $\quad(1) \Rightarrow(2)$ Choose by uniform continuity of $\varphi$ a number $\delta>0$ such that whenever $\|x-w\| \leqslant \delta$, then $|\varphi(x)-\varphi(w)|<1$. Fix $k \in Z^{+}$and choose $r>0$ such that $\{x: \varphi(x) \geqslant r\} \subset C_{k}$. Then choose $n>k$ such that $\{x: \varphi(x) \geqslant r+1\} \supset C_{n}$. If $w \in C_{n}$ and $x \notin C_{k}$, we have $\varphi(w)-\varphi(x) \geqslant(r+1)-r=1$ and so $\|x-w\|>\delta$. We conclude that $\left(C_{n}+\delta U\right) \cap C_{k}^{c}=\emptyset$, as required.

(2) $\Rightarrow(3)$ Choose $j \in Z^{+}$such that $j \delta>r$, and then by repeatedly applying (2) choose a positive integer $n_{0}>k$ such that $C_{k} \supset C_{n_{0}}+j \delta U$. For each $n>n_{0}$ we have $C_{n} \subset\left\{x: d\left(x, C_{k}^{c}\right) \geqslant r\right\}$, as required.

(3) $\Rightarrow$ (4) By passing to a subsequence, we may assume that $C_{1} \neq X$ and that for each $n \in Z^{+}, C_{n+1} \subset B_{-1}\left(C_{n}\right)$. For each $n \in Z^{+}$, define $f_{n}: X \rightarrow[0, \infty)$ by

$$
f_{n}(x)=\max \left\{n, d\left(x, C_{n}^{c}\right)\right\}=\max \left\{n,-\Delta\left(x, C_{n}\right)\right\}
$$

where $\Delta\left(\cdot, C_{n}\right)$ is the Hirart-Urruty functional. Since $\Delta\left(\cdot, C_{n}\right)$ is convex and 1-Lipschitz, each $f_{n}$ is quasi-concave and is 1-Lipschitz. Now let $\varphi=\inf _{n \in Z^{+}} f_{n}$. Then 
$\varphi$ is quasi-concave, and as $\varphi$ is the infimum of a family of 1-Lipschitz functions that is somewhere finite, $\varphi$ is 1-Lipschitz.

We now verify that $\varphi$ is a forcing function for $\left\langle C_{k}\right\rangle$. First, if $x \notin C_{k}$, then $d\left(x, C_{k}^{c}\right)=0$ and so $\varphi(x) \leqslant f_{k}(x)=k<k+1$. This shows that $\{x: \varphi(x) \geqslant k+1\} \subset$ $C_{k}$. On the other hand, suppose $x \in C_{2 k}$. If $n<2 k$, then $d\left(x, C_{n}^{c}\right) \geqslant 2 k-n$ and so

$$
f_{n}(x) \geqslant \max \{n, 2 k-n\} \geqslant k \quad(1 \leqslant n<2 k) .
$$

On the other hand, if $n \geqslant 2 k$ then $f_{n}(x) \geqslant 2 k>k$. As a result, $\varphi(x)=\inf _{n \in Z^{+}} f_{n}(x) \geqslant k$ which shows that $\{x: \varphi(x) \geqslant k\} \supset C_{2 k}$.

(4) $\Rightarrow$ (1) This is trivial.

From Theorem 4.4, no Lipschitz forcing function exists for the following convex mode of convergence to infinity in the plane:

$$
\left\langle\left\{\left(\alpha_{1}, \alpha_{2}\right): \alpha_{1}>0, \alpha_{2}>0 \text { and } \alpha_{1} \alpha_{2} \geqslant k\right\}\right\rangle \text {. }
$$

\section{Positive convergence to infinity}

Let $C$ be a closed pointed cone in a normed linear space $X$ with nonempty interior. Recall from Section 1 that positive convergence to infinity as determined by $C$ is represented by the sequence $\left\langle k x_{0}+C\right\rangle$ where $x_{0}$ is an arbitrary point of $\operatorname{int} C$. As we shall set in Proposition 5.4 below, the bounded sets with respect to positive convergence to infinity are those that fit the following:

Definition: Let $X$ be a normed linear space with positive cone $C$. We call a subset $A$ of $X$ iso-bounded above with respect to $C$ provided $A-C \neq X$.

This concept was considered by Borwein, Penot and Thera [3] in their study of convex mappings into an ordered topological vector space. A subset $A$ is not isobounded above provided for each $x \in X$ there exists $a \in A$ such that $a \geqslant x$, and in this case the authors wrote isosup $A=\infty$. Of course, a subset $A$ of $X$ is called order bounded above if for some $x_{0} \in X, A \subset x_{0}-C$. If no such $x_{0}$ exists, then it is standard to write $\sup A=\infty$. In this section, we display natural forcing functions for positive convergence to infinity and for the non-convex mode of convergence to infinity whose bounded sets are those that are order bounded above. It turns out that there is no need to assume that the cone $C$ is pointed. only that int $C \neq \emptyset$ and $C \neq X$. We shall not explicitly state that $C \neq X$ in the sequel, but this is to be understood.

The analysis is facilitated by introducing two auxiliary 1-Lipschitz functions associated with the cone $C$ :

$$
g(x)=\inf \left\{y(x): y \in C^{*} \cap S^{*}\right\}
$$


and

$$
h(x)=\sup \left\{y(x): y \in C^{*} \cap S^{*}\right\} .
$$

Obviously, $g$ is concave and positively homogeneous, whereas $h$ is convex and positively homogeneous. Let us first express these in terms of the Hirart-Urruty functional.

LEMMA 5.1. Let $C$ be a closed convex cone in a normed linear space $X$ with nonempty interior. Then $h=\Delta(\cdot,-C)$ and $g=-\Delta(\cdot, C)$. In particular, $h(x) \leqslant 0$ if and only if $x \in-C$ and $g(x) \geqslant 0$ if and only if $x \in C$.

Proof: We just verify the first statement. Since $s(y,-C)$ is finite if and only if $y \in(-C)^{\circ}=C^{*}$, we have for $x \in X$

$$
\begin{aligned}
\Delta(x,-C) & =\sup \left\{y(x)-s(y,-C): y \in S^{*}\right\} \\
& =\sup \left\{y(x)-0: y \in C^{*} \cap S^{*}\right\}=h(x) .
\end{aligned}
$$

That $h(x) \leqslant 0$ if and only if $x \in-C$ is a restatement of the Bipolar Theorem; alternatively, it follows from the formula $\Delta(x,-C)=d(x,-C)-d\left(x,(-C)^{c}\right)$.

We first show that each translate of $C$ by an element of int $C$ contains a superlevel set of $g$ and vice versa, whereas each such translate of $-C$ is contained in a sublevel set of $h$ and vice versa. Of course, these inclusion properties separately force $C$ to have nonempty interior, and so there is no hope to obtain such properties more generally. These facts allow us to characterise usefully upper boundedness in several ways. We then use $g$ (respectively $h$ ) to produce a metric uniformly equivalent to the metric determined by the initial norm whose bounded sets coincide with the iso-upper bounded sets (respectively, order upper bounded sets).

LEMMA 5.2. Let $C$ be a closed convex cone in a normed linear space $X$ with nonempty interior, and let $x_{0} \in \operatorname{int} C$ with $\left\|x_{0}\right\|=1$. Then for each $k \in Z^{+}$there exist integers $n$ and $j$ such that

(1) $k x_{0}+C \supset B_{-n}(C)$;

(2) $B_{-k}(C) \supset j x_{0}+C$.

ProOF: For (1), we show that the choice $n=k$ does the job. We first observe that because $g$ is 1 -Lipschitz and $g(\theta)=0$, we have $g\left(-x_{0}\right) \geqslant-1$. Also, since $g$ is concave and positively homogeneous, $g$ is super-additive. Thus, if $x \in C$ and $d\left(x, C^{c}\right) \geqslant k$. Lemma 5.1 yields

$$
g\left(x-k x_{0}\right) \geqslant g(x)+g\left(-k x_{0}\right) \geqslant g(x)-k \geqslant k-k=0
$$

By Lemma 5.1 again, we have $x-k x_{0} \in C$, and so $x \in k x_{0}+C$. For (2), choose $j \in Z^{+}$such that $j d\left(x_{0}, C^{c}\right) \geqslant k$. Now if $x \in j x_{0}+C$, then as $x-j x_{0} \in C$, the 
definition of $C^{*}$ gives

$$
\begin{aligned}
g(x) & =\inf \left\{y\left(j x_{0}\right)+y\left(x-j x_{0}\right): y \in C^{*} \cap S^{*}\right\} \\
& \geqslant \inf \left\{y\left(j x_{0}\right): y \in C^{*} \cap S^{*}\right\} \\
& =j g\left(x_{0}\right)=j d\left(x_{0}, C^{c}\right) \geqslant k .
\end{aligned}
$$

By Lemma 5.1, this shows that $B_{-k}(C) \supset j x_{0}+C$, and completes the proof.

Lemma 5.3. Let $C$ be a closed convex cone in a normed linear space $X$ with nonempty interior, and let $x_{0} \in \operatorname{int} C$ with $\left\|x_{0}\right\|=1$. Then for each $k \in Z^{+}$there exist integers $n$ and $j$ such that

(1) $n x_{0}-C \supset B_{k}(-C)$;

(2) $B_{j}(-C) \supset k x_{0}-C$.

Proof: For (1), choose $n$ such that $g\left(n x_{0}\right)=n g\left(x_{0}\right)>k$. Now if $x \in B_{k}(-C)$, then by Lemma 5.1, we have $h(x) \leqslant k$. Thus, for each $y \in C^{*}$, we. have

$$
y\left(n x_{0}-x\right)=y\left(n x_{0}\right)-y(x) \geqslant g\left(n x_{0}\right)-h(x) \geqslant 0 .
$$

By the Bipolar Theorem, $n x_{0}-x \in C$ and so $n x_{0}-C \supset B_{k}(-C)$.

For (2), because $h$ is 1-Lipschitz and $h(\theta)=0$, we have $h\left(k x_{0}\right) \leqslant k\left\|x_{0}\right\|=k$ and if $x \in k x_{0}-C$, then $h(x) \leqslant h\left(k x_{0}\right)$. Thus, $k x_{0}-C \subset B_{k}(-C)$.

Proposition 5.4. Let $X$ be a normed linear space ordered by a closed cone $C$ with nonempty interior. Let $A$ be a subset of $X$. The following conditions are equivalent:

(1) $A$ is iso-bounded above;

(2) for each $x_{0} \in \operatorname{int} C$ there exists $k \in Z^{+}$such that $A \cap\left(k x_{0}+C\right)=\emptyset$;

(3) there exist $n \in Z^{+}$such that $B_{-n}(C) \cap A=\emptyset$;

(4) $\sup _{x \in A} g(x)<\infty$.

Proof: $(1) \Rightarrow(2)$ Since $A$ is iso-bounded above, there exists $x \in X$ such that $A \cap(x+C)=\emptyset$. Fix $x_{0} \in \operatorname{int} C$ and choose $k \in Z^{+}$so large that $x_{0}-(x / k) \in C$. Since $C=k C$, we have $k x_{0} \in x+C$, and so $k x_{0}+C \subset x+C+C=x+C$. As a result, we have $A \cap\left(k x_{0}+C\right)=\emptyset$.

(2) $\Rightarrow$ (3) Fix $x_{0} \in \operatorname{int} C$ with $\left\|x_{0}\right\|=1$ and choose $k \in Z^{+}$such that $A \cap$ $\left(k x_{0}+C\right)=\emptyset$. By Lemma 5.2 we can find a positive integer $n$ such that $k x_{0}+C \supset$ $B_{-n}(C)$, and so $B_{-n}(C) \cap A=\emptyset$.

(3) $\Rightarrow$ (4) If $x \notin B_{-n}(C)$, then either $x \notin C$ or $x \in C$ and $d\left(x, C^{c}\right)<n$. Since $g(x)<0$ for $x \notin C$, and $g(x)=d\left(x, C^{c}\right)$ for $x \in C$, we have

$$
\sup _{x \in A} g(x) \leqslant \max \left\{0, \sup _{x \in A \cap C} g(x)\right\} \leqslant n .
$$


(4) $\Rightarrow$ (1) Choose $k \in Z^{+}$such that $\sup _{x \in A} g(x)<k$. Fix $x_{0} \in \operatorname{int} C$ with $\left\|x_{0}\right\|=1$. By Lemma 5.2 there exists $j \in Z^{+}$such that $B_{-k}(C) \supset j x_{0}+C$, and it follows that $j x_{0} \notin A-C$. Thus, $A$ is iso-bounded above.

Note that the equivalence of conditions (1) and (4) says that $x \rightarrow \max \{0, g(x)\}=$ $d\left(x, C^{c}\right)$ is a (quasi-concave) forcing function for positive convergence to infinity. Essentially, this is the assertion of [3, Lemma 1.1] established using the machinery of order-unit seminorms $[4$, p.230] under the assumption of normality of $C$. But here we do not assume normality, and do not renorm the space.

The proof of the following analogue for order upper boundedness based on Lemma 5.3 is left to the reader. The equivalence of conditions (1) and (2) says that the sets which are order bounded above are the bounded sets determined by the mode of convergence to infinity $\left\langle\left(k x_{0}-\operatorname{int} C\right)^{c}\right\rangle$, and the equivalence of (1) and (4) says that $x \rightarrow d(x,-C)$ is a compatible forcing function.

Proposition 5.5. Let $X$ be a normed linear space ordered by a closed cone $C$ with nonempty interior. Let $A$ be a subset of $X$. The following conditions are equivalent:

(1) $A$ is order bounded above;

(2) for each $x_{0} \in \operatorname{int} C$ there exists $k \in Z^{+}$such that $A \subset k x_{0}-C$;

(3) there exist $n \in Z^{+}$such that $B_{n}(-C) \supset A$;

(4) $\sup _{x \in A} h(x)<\infty$.

Upper boundedness of the functions $g$ and $h$ on a convex set may be recast dually as follows.

PRoposition 5.6. Let $X$ be a normed linear space ordered by a closed cone $C$ with nonempty interior. Let $A$ be a convex subset of $X$. Then:

(1) $A$ is iso-bounded above if and only if for some $\lambda>0$ we have $(\lambda A)^{\circ} \cap$ $\left(C^{*} \cap S^{*}\right) \neq \emptyset$;

(2) $A$ is order bounded above if and only if for some $\lambda>0$ we have $(\lambda A)^{\circ} \supset$ $C^{*} \cap S^{*}$.

Proof: For (1), suppose $A$ is iso-bounded above. Let $x_{0} \in \operatorname{int} C$, and choose $k \in Z^{+}$such that $A \cap\left(k x_{0}+C\right)=\emptyset$. By the separation theorem, there exists $y \in S^{*}$ such that

$$
\sup \{y(a): a \in A\} \leqslant \inf \left\{y(x): x \in k x_{0}+C\right\} .
$$

Clearly, $y \in C^{*}$, else $\inf \left\{y(x): x \in k x_{0}+C\right\}=-\infty$. Choosing $\lambda>0$ such that $\lambda \sup \{y(a): a \in A\} \leqslant 1$, we see that $y \in(\lambda A)^{\circ} \cap\left(C^{*} \cap S^{*}\right)$. Conversely, if $y \in$ $(\lambda A)^{\circ} \cap\left(C^{*} \cap S^{*}\right)$, then $\sup _{x \in A} y(x) \leqslant \lambda^{-1}$ and so $\sup _{x \in A} g(x) \leqslant \lambda^{-1}$. By Proposition 5.4, 
$A$ is iso-bounded above.

For (2), suppose $A$ is order upper bounded. Choose by Proposition $5.5 \lambda>0$ such that $\sup h(x) \leqslant \lambda^{-1}$. Then for each $y \in C^{*} \cap S^{*}$, we have $s(y, \lambda A) \leqslant 1$ and so $y \in(\lambda A)^{\circ}$. Conversely, if for some $\lambda>0$ we have $(\lambda A)^{\circ} \supset C^{*} \cap S^{*}$, then for each $y \in C^{*} \cap S^{*}$ we have $\sup _{x \in A} y(x) \leqslant \lambda^{-1}$ so that

$$
\begin{aligned}
\sup _{y \in C^{*} \cap S^{*}} \sup _{x \in A} y(x) & =\sup _{x \in A} \sup _{y \in C^{*} \cap S^{*}} y(x) \\
& =\sup _{x \in A} h(x) \leqslant \lambda^{-1}
\end{aligned}
$$

This proves that $A$ is order upper bounded.

For completeness, we present two remetrisation theorems in the spirit of [12]. As the proofs are almost identical modulo replacing $g$ by $h$, we only provide one for the first.

THEOREM 5.7. Let $X$ be a normed linear space ordered by a closed cone $C$ with nonempty interior. Then the metric $\rho$ on $X$ defined by

$$
\rho(x, w)=\min \{\|x-w\|, 1\}+\left|d\left(x, C^{c}\right)-d\left(w, C^{c}\right)\right|
$$

is uniformly equivalent to the metric of the initial norm on $X$, and the $\rho$-bounded subsets coincide with the iso-upper bounded subsets of $X$ as determined by $C$.

Proof: Denote the metric determined by the norm by $d$. By the uniform continuity of distance functionals, $\rho$ is uniformly equivalent to $d$. Since $d\left(\theta, C^{\mathrm{c}}\right)=0$, Lemma 5.1 tells us that $\rho(\theta, x)=\min \{\|x\|, 1\}+\max \{0, g(x)\}$, where as usual

$$
g(x)=\inf \left\{y(x): y \in C^{*} \cap S^{*}\right\} .
$$

Thus, if $A \subset\{x: \rho(x, \theta) \leqslant r\}$, then for each $a \in A$, we have $\max \{0, g(a)\} \leqslant r$ and so $\sup g(a) \leqslant r$. Thus, $A$ is iso-bounded above. Conversely, if $A$ is iso-bounded above, $a \in A$

then for some $r>0, \sup _{a \in A} g(x) \leqslant r$ and so $A \subset\{x: \rho(x, \theta) \leqslant r+1\}$.

ThEOREM 5.8. Let $X$ be a normed linear space ordered by a closed cone $C$ with nonempty interior. Then the metric $\rho$ on $X$ defined by

$$
\rho(x, w)=\min \{\|x-w\|, 1\}+|d(x,-C)-d(w,-C)|
$$

is uniformly equivalent to the metric of the initial norm, and the $\rho$-bounded subsets coincide with the order upper bounded subsets of $X$ as determined by $C$. 


\section{REFERENCES}

[1] G. Beer, Topologies on closed and closed convex sets (Kluwer Academic Publishers, Dordrecht, Holland, 1993).

[2] G. Beer, 'On metric boundedness structures', Set-Valued Anal. (to appear).

[3] J. Borwein, J.-P. Penot and M. Thera, 'Conjugate convex operators', J. Math. Anal. Appl. 102 (1984), 399-414.

[4] K. Deimling, Nonlinear functional analysis (Springer-Verlag, Berlin, Heidelberg, New York, 1985).

[5] J. Dugundji, Topology (Allyn and Bacon, Boston, 1966).

[6] C. Franchetti and I. Singer, 'Best approximation by elements of caverns in normed linear spaces', Boll. Un. Mat. Ital. (5) 17 (1980), 33-43.

[7] H. Hadwiger, Vorlesungen über Inhalt, Oberfläche und Isoperimetrie (Springer-Verlag, Berlin, Heidelberg, New York, 1957).

[8] J.-B. Hiriart-Urruty, 'Tangent cones, generalized gradients, and mathematical programming in Banach spaces', Math. Oper. Res. 4 (1979), 79-97.

[9] J.-B. Hiriart-Urruty, 'New concepts in nondifferentiable programming', Bull. Soc. Math. France Mem. 60 (1979), 57-85.

[10] J.-B. Hiriart-Urruty and C. Lemaréchal, Convex analysis and minimization algorithms I (Springer-Verlag, Berlin. Heidelberg, New York, 1993).

[11] R. Holmes, A course in optimization and best approximation, Lecture Notes in Mathematics 257 (Springer-Verlag, Berlin, Heidelberg, New York, 1972).

[12] S.-T. Hu, Introduction to general topology (Holden-Day, San Francisco, 1966).

[13] B. Lemaire, 'Duality in reverse convex optimization', SIAM J. Optimization (to appear).

[14] J. Sangwine-Yager, 'Bonnesen-style inequalities for Minkowski relative geometry', Trans. Amer. Math. Soc. 307 (1988), 373-382.

[15] M. Sion, 'On general minimax theorems', Pacific J. Math. 8 (1958), 171-176.

[16] J. Van Tiel, Convex analysis (J. Wiley and Sons, New York, 1984).

Department of Mathematics

California State University

Los Angeles CA 90032

United States of America 